\title{
Research on the Numerical Simulation of Ship Bow Flare Slamming
}

\author{
Xiaopeng GAO \& Xin GAO \\ Naval University of Engineering, Wuhan, Hubei, China
}

\begin{abstract}
The numerical simulation method of 3D-ship bow flare slamming based on finite volume method is discussed in this paper. A three-Dimension coupling finite element model including air, water and ship bow was built up. The fluid-structure interaction is simulated using general coupling algorithm; Euler formulation is used for the fluid domain while Lagrangian formulation is used for the structure one, ship bow flare slamming pressure distribution in the side position is analyzed. The result shows that the numerical simulation method used here is reasonable to calculate the ship bow flare slamming problem, and an optimization method for ship bow linear designing could be provided.
\end{abstract}

KEYWORD: Flare slamming; Fluid-structure interaction; Pressure distribution

\section{INTRUDUCTION}

When the ship is sailing in the waves, the bottom of the course of the campaign is not necessarily beyond the water, but when waves impact the forward part of the flare region, it also produces a larger pulse hydrodynamic, called flare slamming[1]. The flare slamming has some characteristics, like smaller slamming pressure, the role of larger surface area and longer slamming process duration. Determining the flare slamming pressure of a ship is essential to the hull structure design. Therefore, for such as container ships, we must consider the impact of the slamming in the early design process.

For the structures into water slamming process, abroad Von Karma[2], Wagner[3] and many other scholars studied deeply in the theory. At home Chen Zhen[4] used a theoretical means which combined three-dimensional surface of the hull impact pressure calculation method with ship seakeeping forecast to forecast large container broadside flare slamming pressure.

However, due to complex structures, especially for ship hull, the process of into water is a nonlinear, unsteady problem and it has very complex physical phenomena. A lot of the essential characteristics and physical mechanism of the slamming phenomenon is difficult to interpret with theory. The theoretical analysis is restricted by a variety of factors, such as the geometry of an object, the speed of into the water and non-linear free boundary conditions. In recent years, the rapid development of the computer allows scholars to study the structure of slamming through numerical simulation. Verhagen[5], Jin Fusheng[6] and others used the finite difference method. Zhao \& Faltisen[7] used the boundary element method, LUO Han-bing[8] and others used the explicit finite element method, Xu Fei[9] used Smoothed Particle Hydrodynamics(SPH) method, and YU Qian, ZHANG Huai-xin[10] used MovingParticle Semi-Implicit Method ,but more than most scholars, working methods mainly confined to twodimensional simulation.

This paper applies the transient dynamics software MSC.Dytran to simulate a 3D ship bow model, considering factors such as atmospheric pressure and gravity, and calculate the flare slamming pressure under different speed and analyze the slamming pressure distribution. It could lay the foundation for the follow-up linear design of the ship bow.

\section{GETTING STARTED}

In this paper, a general fluid-structure interaction algorithm is used, namely Lagrangian finite element method to solve the solid model; Eulerian finite volume method for solving fluid model.

\subsection{Lagrangian finite element method}

MSC.Dytran is the high-end software in the transient dynamics flow-solid coupling field. It applies 
explicit time integration method in the time domain. Current time step is $n$, explicit integration method rewrites the differential equations of motion:

$$
\begin{aligned}
& M a_{n}+C v_{n}+K d_{n}=F_{n}^{e x t} \\
& M a_{n}=F_{n}^{e x t}-F_{n}^{i n t} \\
& F_{n}^{i n t}=C v_{n}+K d_{n}
\end{aligned}
$$

Where $F_{n}{ }^{e x t}$ is outside load vector; $F_{n}{ }^{i n t}$ is internal force vector.

Acceleration is obtained by the inverse of the mass matrix and multiplied by the remaining force vector $F_{n}^{\text {residual. }}$

$$
\begin{aligned}
& F_{n}^{\text {residual }}=F^{\text {ext }}-F^{\text {int }} \\
& a_{n}=M^{-1} F_{n}^{\text {residual }}
\end{aligned}
$$

If the element mass distributes on the node, $M$ will become a diagonal matrix, named as a centralized mass matrix. Linear equations will become a series of independent linear equations about the various degrees of freedom, which can be calculated node acceleration:

$$
a_{n i}=F_{n i}^{\text {residual }} / M_{i}
$$

If it is assumed that the acceleration is constant within a time step, the central difference method could be used on the time marching:

$$
\begin{aligned}
& v_{n+1 / 2}=v_{n-1 / 2}+a_{n}\left(\Delta t_{n+1 / 2}+\Delta t_{n-1 / 2}\right) / 2 \\
& d_{n+1}=d_{n}+v_{n+1 / 2} \Delta t_{n+1 / 2}
\end{aligned}
$$

Explicit integration method does not need to do matrix decomposition and therefore has a very high computational efficiency.

\subsection{Eulerian finite volume method}

Under the case of the initial conditions are known, the control equations[11] is used to solve each Euler unit's density, velocity, specific internal energy and the pressure at a time step. It is assumed that various physical parameters are known at time $t_{n}$, so the velocity of cell boundary will be obtained by the linear interpolation of velocity on the centroid of adjacent cells.

$$
u_{b}=1 / 2\left(u_{1}+u_{2}\right)
$$

The flow of mass, momentum and energy through the cell surface will be obtained:

$$
\begin{aligned}
& \Delta M=\rho_{2} \Delta V \\
& \Delta M \mathrm{om}=\rho_{2} u_{2} \Delta V \\
& \Delta T E=\rho_{2}\left(e_{t}\right) \Delta V
\end{aligned}
$$

Where $\rho_{2}$ is adjacent cell density; $e_{t}$ is the total energy of the element quality; $T$ is the surface force of unit area in the boundary; $\Delta V$ is the surface volume flow through the unit within a time step from $t_{n}$ to $t_{n+1}$. By using the single-point Gaussian integral and control equations, the physical quantity value (density, velocity, and internal energy) of unit centroid can be solved at the time $t_{n}$ and the change volume relationship will also be obtained from $t_{n}$ to $t_{n+1}$. According to the material constitutive relations, the pressure value can further be calculated.

\subsection{Lagrange - Euler fluid-structure interaction}

With a layer of coupling plane defined by the Lagrangian model and a coupled relationship created, the transmitters between the Lagrange part and Euler part will be produced. Lagrangian part accepts the load directly from the coupling surface. The Euler's part acts the surface as a flow field boundary, and puts stress of Euler unit to the coupling surface, which caused the deformation of the Lagrangian unit. Mainly through three-step coupling calculation[12]: (1) The coupling calculation step; (2) transport step; (3) impulse step. The coupling step calculates intersecting situation of the coupling surface with unit. The transport step is responsible for transferring the quality of unit and intersecting variable with quality. The impulse further adds contribution of the pressure wave spreading in a grid.

\section{CALCULATION MODEL}

Taking the bow of a container ship for example, the flare slamming pressure of ship bow at the broadside area will be calculated. The ship's master scale data is shown in Table 1, and the bow cross section line is shown in Figure 1.

Table 1. The main scale data.

\begin{tabular}{|l|l|}
\hline Main scale & Figure \\
\hline Captain & $159.10 \mathrm{~m}$ \\
\hline Breadth & $26.50 \mathrm{~m}$ \\
\hline Molded depth & $13.30 \mathrm{~m}$ \\
\hline Design Draft & $8.20 \mathrm{~m}$ \\
\hline
\end{tabular}

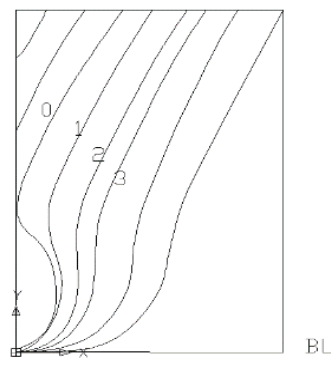

Figure 1. Cross-sectional chart. 
On the basis of the establishment of threedimensional bow model, the slamming problems of bow will be calculated numerically by the MSC.Dytran software. In the simulation process, as shown in Figure 2, the length of the computational domain size is 4 times of bow length, its width is 5 times of the width of the bow portion, its height is 7 times of the height of the bow portion and nonreflective boundary conditions is applied to ensure that propagation of the pressure wave in the flow field will not affect slamming area.

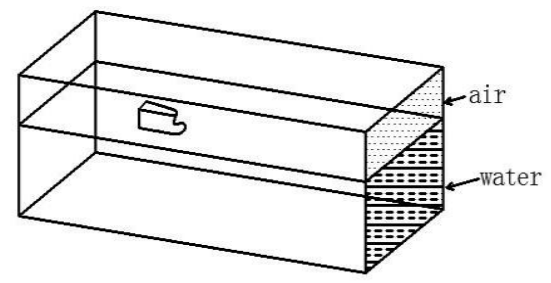

Figure 2. Computational domain diagram.

Euler region is meshed with ranging density grid. In the close location of the bow model structure, grid is more frequent, and far from the bow model structure, it will be rougher. Rational arrangement of Euler grid in the flow field area can effectively improve the computational efficiency.

The bow model structure is divided by Lagrange element grid, with rigid material. The air and water are used Euler unit meshing. Air unit is filled with material which has compressible ideal gas constitutive relation and water unit is filled with material which has non-sticky, compressible linear fluid constitutive relation. The figure 3 is the local meshing circum-stances of the computational model.

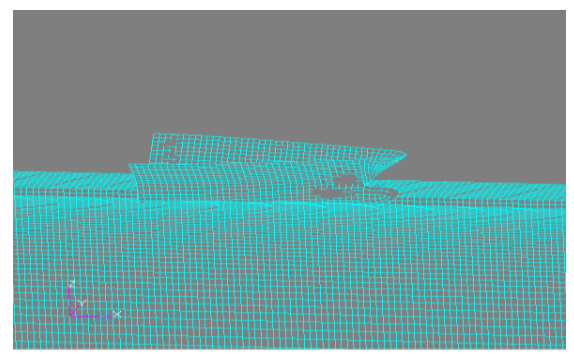

Figure 3. Local meshing.

The entire closed outer surface of bow model is de-fined as the fluid-structure interaction surface. General coupling algorithms (General coupling) is used to calculate the interaction between the fluid and the bow model structure. The parameters set of the calculation model can be seen from reference [13].

\section{RESULTS AND ANALYSIS}

The bow model is calculated in the $5 \mathrm{~m}$ wave height sea conditions and under the $10.0 \mathrm{kn}, 15.0 \mathrm{kn}, 20.0 \mathrm{kn}$ different speed. According to the linear characteristics of the ship bow, in the region of 0 to 3 stations and 9 12m waterline the flare slamming pressure extreme results of ship's rail are shown in Table 2.

Table 2. The flare slamming extreme pressure $\left(\times 10^{5} \mathrm{~Pa}\right)$.

\begin{tabular}{|c|c|c|c|c|c|}
\hline Velocity/kn & WL/m & $\begin{array}{c}0 \\
\text { Station }\end{array}$ & $\begin{array}{c}1 \\
\text { Station }\end{array}$ & $\begin{array}{c}2 \\
\text { Station }\end{array}$ & $\begin{array}{c}3 \\
\text { Station }\end{array}$ \\
\hline \multirow{4}{*}{10.0} & 9 & 0.590 & 0.505 & 0.481 & 0.454 \\
\cline { 2 - 6 } & 10 & 0.618 & 0.535 & 0.500 & 0.463 \\
\cline { 2 - 6 } & 11 & 0.639 & 0.543 & 0.506 & 0.464 \\
\cline { 2 - 6 } & 12 & 0.649 & 0.557 & 0.519 & 0.476 \\
\hline \multirow{4}{*}{15.0} & 9 & 0.599 & 0.514 & 0.489 & 0.463 \\
\cline { 2 - 6 } & 10 & 0.624 & 0.546 & 0.508 & 0.474 \\
\cline { 2 - 6 } & 11 & 0.646 & 0.559 & 0.522 & 0.476 \\
\cline { 2 - 6 } 20.0 & 12 & 0.657 & 0.568 & 0.534 & 0.485 \\
\hline \multirow{5}{*}{} & 9 & 0.613 & 0.530 & 0.509 & 0.489 \\
\cline { 2 - 6 } & 10 & 0.635 & 0.561 & 0.515 & 0.496 \\
\cline { 2 - 6 } & 11 & 0.657 & 0.581 & 0.544 & 0.500 \\
\cline { 2 - 6 } & 12 & 0.668 & 0.597 & 0.565 & 0.514 \\
\hline
\end{tabular}

\subsection{Flare slamming pressure distribution along the ship direction}

Figure 4 and Figure 5 is the slamming extreme pressure changes along the direction of the captain under the $5 \mathrm{~m}$ wave height at different speeds, in the bow of $9 \mathrm{~m}$ waterline and the $12 \mathrm{~m}$ waterline.

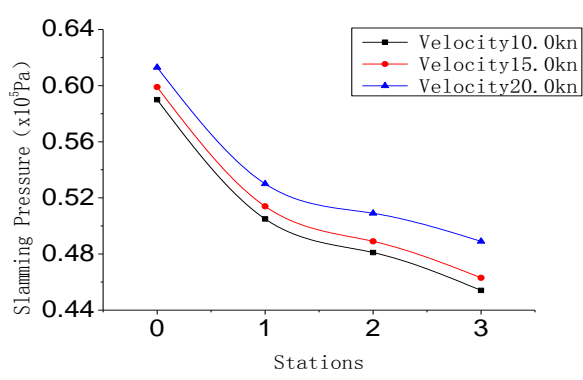

Figure $4.9 \mathrm{~m}$ waterline slamming pressure.

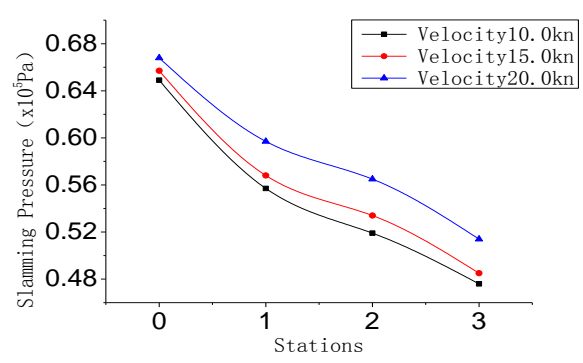

Figure 5. 12m waterline slamming pressure.

As can be seen from the above chart, under the $5 \mathrm{~m}$ wave heights, the flare slamming extreme pressure gradually decreases at the height of the same water-line, along the direction of the bow to the stern. 
Above slamming pressure trends are determined mainly by the hull surface linear characteristics, that float angle gradually decreases from 0 to 3 stations, and thus, according to Wagner's classic slamming theory shows that the flare slamming pressure gradually decreases.

\subsection{Flare slamming pressure along the side height direction distribution}

Figure 6 and Figure 7 respectively is the slamming extreme pressure changes along the height direction under the $5 \mathrm{~m}$ wave height at different speeds, in the bow of 0 station and 3 station.

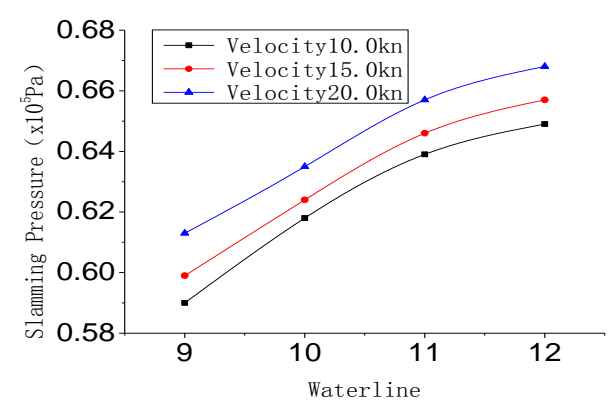

Figure 6. Slamming pressure at 0 station.

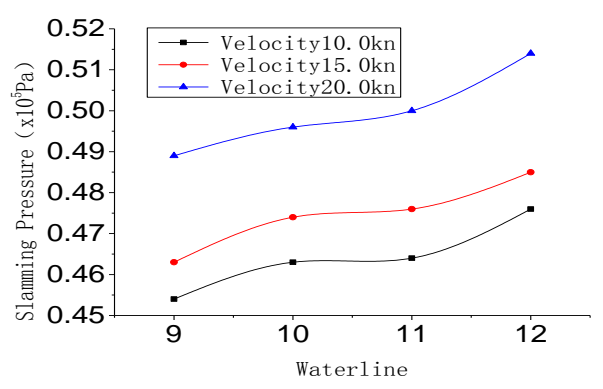

Figure 7. Slamming pressure at 3 station.

Seen from Figures 6 and 7, in the same wave height, the variation of flare slamming extreme pressure along the height direction is that flare slamming pressure increases with the increased height from the waterline.

This is because a broadside linear flare angle increases gradually with the height from the waterline to in-crease in the bow area, and thus by Wagner slamming theory shows the flare slamming pressure in-creases with increasing height.

\section{SUMMARY}

For the flare slamming problems of threedimensional structure, the theoretical research has more limitations. In this paper, by the simulation of flare slamming pressure of the three-dimensional bow model, the following conclusions can be drawn:

1) Through the simulation, this analysis proves flare slamming pressure distribution meets the classic Wagner slamming theory, thus proving the feasibility of the numerical simulation method to study the three-dimensional slamming problems.

2) The flare slamming pressure of ship bow has a closer relationship with the hull surface line type. The maximum flare slamming pressure generally appears in the 0 to 1 station in the flare linear region of the bow.

3) For the line type of ship bow likes container ships, along the Captain direction, its flare slamming extreme pressure decreases with the distance increasing from the bow, and in the height direction the flare slamming extreme pressure increases as the distance of the waterline height increases.

\section{REFERENCES}

[1] DAI Yang-shan et al. 2007. Ship Wave Loads. Beijing: National Defense Industry Press.

[2] Von, K.T. 1929. The impact of seaplane floats during landing. Washington DC, USA: National Advisory Committee for Aeronautics, NACA Technical Notes 321.

[3] Wagner, H. 1932. Phenomena associated with impacts and sliding on liquid surfaces. Z Angew Math Mech 12(4): 193-215.

[4] CHEN Zhen et al. 2011. Study on Flare Impact Characteristics of Large Container Ships. Ship \& Ocean Engineering.

[5] VERHAGEN, J.H.G. 1967. The impact of a flat plate on a water surface. Journal of Ship Research 11(4): 211-223.

[6] Jin Fu-sheng. 1992. Into the water shock variational principle and the other. Applied Mathematics and Mechanic 13(6): 543-552.

[7] Zhao, R. \& Faltinsen, O. 1993. Water entry of twodimensional bodies. Journal of Fluid Mechanics 246: 593-612.

[8] LUO Han-bing et al. 2012. Parallel computing simulation of water entry of a 2D-rigid wedge using an explicit finite element method. Journal of Ship Mechanics 08: 907-914.

[9] Xu Fei. 2013. A 2D smoothed particle hydrodynamics study of flared bow slamming. Shanghai: Shanghai Jiao Tong University.

[10] YU Qian \& ZHANG Huai-xin. 2013. Research on water entry of wedge based on the improved MPS method with large eddy simulation. The Ocean Engineering 06:9-15.

[11] Bian Wen-jie et al. 2004. The transient dynamics CAE the solution MSC.Dytran foundation tutorial. Beijing: Peking University Press.

[12] Ding Pei-ran \& Qian Chun. 2006. Nonlinear transient dynamics the analysis-MSC.Dytran theory and application. Beijing: Science Press.

[13] CHEN Zhen \& XIAO Xi. 2005. Simulation Analysis on the Role of Air Cushion in the Slamming of a Flat-Bottom Structure. Journal of Shanghai Jiaotong University 39(5): 670 674. 